\title{
ELASTOMER ACTUATORS: SYSTEMATIC IMPROVEMENT IN PROPERTIES BY USE OF COMPOSITE MATERIALS
}

\author{
Martin Molberg $^{\mathrm{a}, \mathrm{b}^{*}}$, Yves Leterrier ${ }^{\mathrm{b}}$, Christopher J. G. Plummer ${ }^{\mathrm{b}}$, Christiane Löwe ${ }^{\mathrm{a}}$, Dorina M. \\ Opris $^{\mathrm{a}}$, Frank Clemens ${ }^{\mathrm{c}}$, Jan-Anders E. Månson ${ }^{\mathrm{b}}$ \\ a Laboratoy for Functional Polymers, Swiss Federal Laboratories for Material Testing \\ and Research (Empa), CH-8600 Dübendorf, ZH, Switzerland. \\ b Laboratoire de Technologie des Composites et Polymères (LTC), École \\ Polytechnique Fédérale de Lausanne (EPFL), CH-1015 Lausanne, VD, Switzerland \\ c Laboratory for High-Performance Ceramics, Swiss Federal Laboratories for Material \\ Testing and Research (Empa), CH-8600 Dübendorf, ZH, Switzerland.
}

\begin{abstract}
Dielectric elastomer actuators (DEAs) have attracted increasing attention over the last few years owing to their outstanding properties, e.g. their large actuation strains, high energy density, and pliability, which have opened up a wide spectrum of potential applications in fields ranging from microengineering to medical prosthetics. There is consequently a huge demand for new elastomer materials with improved properties to enhance the performance of DEAs and to overcome the limitations associated with currently available materials, such as the need for high activation voltages and the poor long-term stability. The electrostatic pressure that activates dielectric elastomers can be increased by higher permittivity of the elastomer and thus may lead to lower activation voltages. This has led us to consider composite elastomeric dielectrics based on thermoplastic elastomers or PDMS, and conductive polyaniline or ceramic (soft doped PZT) powder fillers. The potential of such materials and strategies to counter the adverse effects of increased conductivity and elastic modulus are discussed.
\end{abstract}

Keywords: dielectric elastomer, electroactive polymer, polymer composite

\section{INTRODUCTION}

Implementation of dielectric elastomer actuators (DEAs) in the huge number of potential applications that have been identified to date remains hindered by the lack of optimized dielectric elastomer materials. The materials used at present are acrylates from the VHB series supplied by $3 \mathrm{M}$, various silicones, thermoplastic elastomers and polyurethanes. ${ }^{1}$ Actuators fabricated from these materials require high activation voltages and this severely limits their applicability in industrial products, in spite of their many advantages, including pliability, large actuation strains, and low densities. ${ }^{2}$

The considerable effort over the last few years to develop elastomeric materials with enhanced properties has focused on increasing their dielectric constant ${ }^{3-8}$, based on the formula given by Pelrine ${ }^{9}$, which relates the electrostatic pressure, $p$, to the dielectric constant, $\varepsilon$, of the material and the electric field strength. The strain, $s$, in a thin film actuator is then given by $p$ and the elastic modulus $Y$ according to

$$
\left.s=\frac{p}{Y}=\frac{\varepsilon \varepsilon_{0}}{Y}\left(\frac{V}{d}\right)^{2} \quad \text { (Equation } 1\right)
$$

where $\varepsilon_{0}=8.854 \times 10^{-12} \mathrm{~F} / \mathrm{m}$ is the permittivity of free space, $V$ is the voltage, and $d$ is the thickness of the film.

*martin.molberg@empa.ch; phone +41 4482343 04; www.empa.ch

Electroactive Polymer Actuators and Devices (EAPAD) 2010, edited by Yoseph Bar-Cohen,

Proc. of SPIE Vol. 7642, 76420M - (C) 2010 SPIE · CCC code: 0277-786X/10/\$18 - doi: 10.1117/12.847493 
Strategies to improve the dielectric properties of dielectric elastomers are generally based on the use of fillers, such that the resulting composites combine the mechanical properties of the matrix elastomer with the high permittivity of the filler. High permittivity polymer composites typically contain either ceramic fillers (e.g $\mathrm{BaTiO}_{3}, \mathrm{TiO}_{2}$, lead lanthanum zirconate titanate $(\mathrm{PZT}))^{10}$ or conductive particles (metals, carbon black, nanotubes, or conductive polymers) ${ }^{11}$ or mixtures of both. ${ }^{12,13}$ However, ceramic and conductive fillers exhibit significant drawbacks, namely their high elastic moduli and the reduced electrical breakdown strength of percolating systems. This will be discussed further in what follows in the light of a detailed evaluation of the material properties and actuation performance of composites prepared from TPE and conductive polyaniline (PANI) or ceramic (PZT) as the high dielectric filler, and the extent to which a proper choice of component materials and processing conditions can lead to composites with superior actuation performance.

\section{EXPERIMENTAL}

A styrene-isoprene block copolymer thermoplastic elastomer (TPE) containing aliphatic oils (Betaflex from Revoflex AG) was used as the matrix for the composites. The TPE pellets were purified by dissolution in toluene, filtering and drying.

Polyaniline (PANI) was purchased from Aldrich (emeraldine base, MW 5000) and doped with dodecylbenzene sulfonic acid (DBSA) in a molar ratio of 1:0.5 to give conductivity and (partial) solubility in non-polar solvents. PANI base and DBSA were added to a non-polar solvent, either toluene or tetrahydrofurane (THF), and mixed with a magnetic stirrer for 1 day. The dark blue color of PANI changes to dark green after doping.

The TPE was dissolved in toluene or THF, the doped PANI solution added and the mixture dispersed by ultrasound, first in an ultrasound-bath, and then using a sonicator (Vibracell $750 \mathrm{~W}$ ) for 5 to 10 minutes. Immediately after dispersion, the solvent was removed by a rotary evaporator. The resulting mixture was then dried further in a vacuum oven (50 mbar, 70 ${ }^{\circ} \mathrm{C}$ ) and pressed several times to remove any residual solvent

Films were prepared for dielectric spectroscopy and fabrication of the actuators by first pressing a $25 \mathrm{~mm}$ diameter cylinder of the TPE at $160^{\circ} \mathrm{C}$ and $100 \mathrm{kPa}$, and then further pressing the resulting cylinder for $15 \mathrm{~min}$ between two glass plates to a final thickness of 100 to $200 \mu \mathrm{m}$ at $160{ }^{\circ} \mathrm{C}$ and $250 \mathrm{kPa}$.

To prepare ceramic filled composites, a commercial soft doped lead zirconate-titanate (PZT) powder (EC65, EDO Corporation) was pre-coated with steatric acid (Fluka AG) to avoid agglomeration during mixing conditions. After precoating the powder exhibit a mean grain size of $1.39 \mu \mathrm{m}$ and the specific surface area of $0.90 \mathrm{~m}^{2} / \mathrm{g}$. For these investigation a laser diffraction method (LS230, Beckman-Coulter) and BET (SA3100, Beckman-Coulter) were used. Different amounts of coated PZT powder were added to a $10 \mathrm{wt} \%$ solution of TPE in cyclohexane and stirred for $30 \mathrm{~min}$. In order to avoid sedimentation of the particles the solvent was removed using a rotary evaporator while sonicating in a heated ultrasound bath. Compounds containing 50, 60, 70, $80 \mathrm{wt} \%$ of PZT were produced by this method and further analyzed.

The thicknesses of the films were measured using a precision thickness gauge MT-30 from Heidenhain in which a caliper applies a small force to the specimen $(2.2 \mathrm{~g}$ over a circular area of diameter $6 \mathrm{~mm})$ and the distance between the caliper position and a reference point is detected by a displacement transducer with a resolution of $0.5 \mu \mathrm{m}$.

A precision LCR-meter HP4284 in combination with a dielectric test fixture 16451B using an electrode of $5 \mathrm{~mm}$ diameter was used for dielectric measurements. The permittivity was determined from the measured capacitance $C=$ $\varepsilon \varepsilon_{0} A / d$, where $A$ is the electrode area. The films were slightly compressed during the dielectric constant measurements owing to the weight of the upper electrode, but this also ensured a good contact with the electrode. $d$ was taken to be the distance between the electrodes as determined by using a micrometer gauge. 
For tensile tests, films with thicknesses of about $1.3 \mathrm{~mm}$ were used to ensure sufficient accuracy in the force measurements. The tensile tests were performed using a Zwick Z010 tensile test machine with a crosshead speed of 500 $\mathrm{mm} / \mathrm{min}$. Tensile test specimens with a gauge width of $3 \mathrm{~mm}$ and a gauge length of $30 \mathrm{~mm}$ were prepared by die cutting. An optical deformation sensor (Zwick video extensometer 066975) was used to detect the tensile strain in the specimens based on measurement of the distance between two marker points placed along the axis of the tensile test bar to an accuracy of $5 \mu \mathrm{m}$.

Oscillatory shear tests were performed on an ARES rheometer (Rheometric Scientific) in parallel plate geometry with cylindrical specimens of $25 \mathrm{~mm}$ diameter and $2 \mathrm{~mm}$ in thickness at frequencies between $0.8 \mathrm{mHz}$ and $80 \mathrm{~Hz}$. All the mechanical tests were carried out under controlled standard atmosphere.

For microscopic analysis, sections of the drawn films were prepared using a cryo-ultramicrotome (Ultracut $\mathrm{E}$ from Reichert-Jung) at $-90{ }^{\circ} \mathrm{C}$ and either glass knives for $1 \mu \mathrm{m}$ thick sections from of TPE/PANI, or diamond knives (Diatome) for $0.3 \mu \mathrm{m}$ sections from TPE/PZT. Micrographs were recorded using an Olympus BH-2 optical microscope.

Actuator tests were performed on circular membrane actuators with prestrained films clamped onto a circular frame with an inner diameter of $30 \mathrm{~mm}$. Circular electrodes $(8 \mathrm{~mm}$ diameter) of fine carbon black powder (Ketjenblack) were applied to each side of the film. A TREK Model 5/80 high voltage source served as the power supply for actuator tests. A video camera controlled by Videoextensometer software from Messphysik $\mathrm{GmbH}$, which detects the boundary between the dark electrode area and translucent surrounding area (bright-dark contrast), was used to determine the diameter of the electrode in two orthogonal directions and hence the mean radial strain in the actuators.

\section{RESULTS AND DISCUSSION}

Use of conductive fillers is a promising approach to increasing the dielectric constant of elastomers. It has been shown that the permittivity of composites increases significantly even at low conductive filler concentrations following a power law, as predicted from percolation theory ${ }^{14}$ :

$$
\varepsilon_{\text {comp }} \propto \varepsilon_{m}\left|c-c_{c}\right|^{-b} \quad \text { (Equation 2) }
$$

where $\varepsilon_{c o m p}$ and $\varepsilon_{m}$ are the permittivities of the composite and the matrix respectively, $c$ is the filler concentration and $c_{c}$ is the concentration at the percolation threshold, where a conductive network is established by the fillers.

On the basis of these results, doped PANI was dispersed in TPE. The resulting blends retained the flexibility of the matrix elastomer and exhibited permittivities in excess of 1000 (e.g. 4462 for $25 \mathrm{wt} \%$ ) compared to the value of 2 measured for the unmodified matrix. However, the increase of the permittivity was also accompanied by an increase in conductivity. Experimental values for several composites with different compositions and processing conditions are given in figure 1. An increase of one order of magnitude in the dielectric constant resulted in an increase of conductivity of more than 3 orders of magnitude. Furthermore, the conductivity of the composite depended on the electric field strength (not shown here) which compounds the situation, because high electric fields are required to actuate dielectric elastomers. 


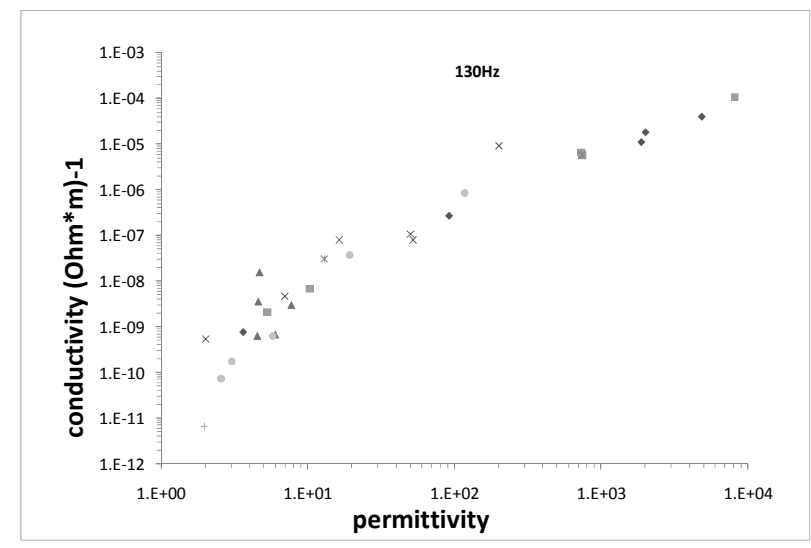

Figure 1: Dielectric constant versus conductivity measured at $130 \mathrm{~Hz}$ for composites of TPE/PANI containing different concentrations of PANI. Different symbols represent different processing procedures.

It was observed that the processing route had a significant impact on the dielectric properties, and this was imputed to differences in the quality of dispersion of the filler particles in the polymer matrix. Slow evaporation of the solvent led to high permittivity but also to high conductivity, whereas faster evaporation using a rotary evaporator led to lower conductivity but also reduced permittivity. A further improvement of the filler dispersion in the matrix was achieved by changing the solvent used in the dispersing process from toluene to THF. Owing to the lower boiling point (higher vapor pressure) of THF, evaporation was faster and therefore the formation of large aggregates of PANI particles was kinetically hindered. Micrographs from thin sections of TPE/PANI prepared by the different processing routes are compared in figure 2. While aggregates of more than 10 to $20 \mu \mathrm{m}$ in diameter were observed for TPE/PANI processed in toluene a better dispersion of PANI particles in the TPE matrix is observed and aggregate diameters were limited to a few $\mu \mathrm{m}$ in TPE/PANI processed in THF.
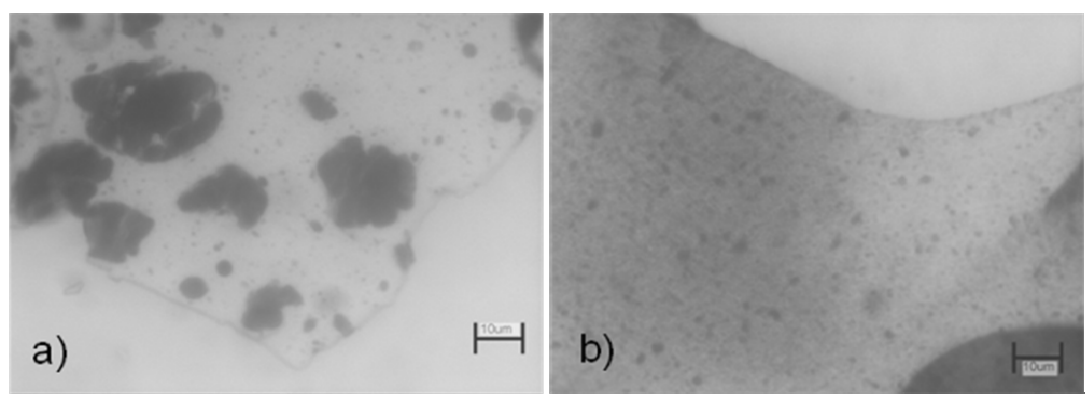

Figure 2: Optical micrographs of sections from TPE/PANI composites a) prepared from toluene ( $22 \mathrm{wt} \%$ PANI) and b) prepared from THF (20 wt $\%$ PANI).

The better dispersion led to a higher permittivity and lower conductivity for a given concentration of PANI. TPE $/ 22 \mathrm{wt} \%$ PANI processed in toluene (cf. figure 2), for example, showed a permittivity of 7 at $130 \mathrm{~Hz}$ and a DC conductivity of 3.9 x $10^{-9} \mathrm{Sm}^{-1}$, whereas TPE/20 wt\% PANI processed in THF gave values of 20.8 and $2.7 \times 10^{-9} \mathrm{Sm}^{-1}$ respectively. However, this range of conductivities remains too high for these materials to be used as dielectric elastomers in DEAs. Similar results were recently reported by Stroyanov et al. ${ }^{15}$ who combined conductive carbon black filler with a thermoplastic elastomer, and found a dramatic decrease in electrical breakdown strength.

An alternative approach to improving the permittivity of polymers is the incorporation of ceramic fillers, and, in particular, ferroelectric ceramics, which are known to show permittivities greater than 1000 . However, a high volume fraction of ceramic is needed to obtain a significant increase in $\varepsilon$. In previous work ${ }^{16}$ on composites of TPE and PZT powder prepared via a thermoplastic extrusion process, surprisingly little influence of the filler concentration was observed on the tensile modulus (a 10 to $15 \%$ increase in the elastic modulus at $100 \%$ strain at the highest filler levels 
investigated of $81 \mathrm{wt} \%$ ). This encouraging result led to further investigations of the use of these materials as dielectric elastomers. To improve the dispersion of the PZT in the matrix the particles were mixed with the elastomer in cyclohexane as described in the experimental section. The quality of dispersion was analyzed using optical micrographs from thin sections of TPE/PZT films, examples of which are shown in figure 3. While the smallest particles had diameters of about $1 \mu \mathrm{m}$, a number of agglomerates with diameters exceeding $10 \mu \mathrm{m}$ were also present. The size of the agglomerates also apparently increased with increasing filler concentration, which is an important consideration given that it is generally necessary to reduce the thickness of the films as much as possible in DEAs in order to reduce the driving voltage.

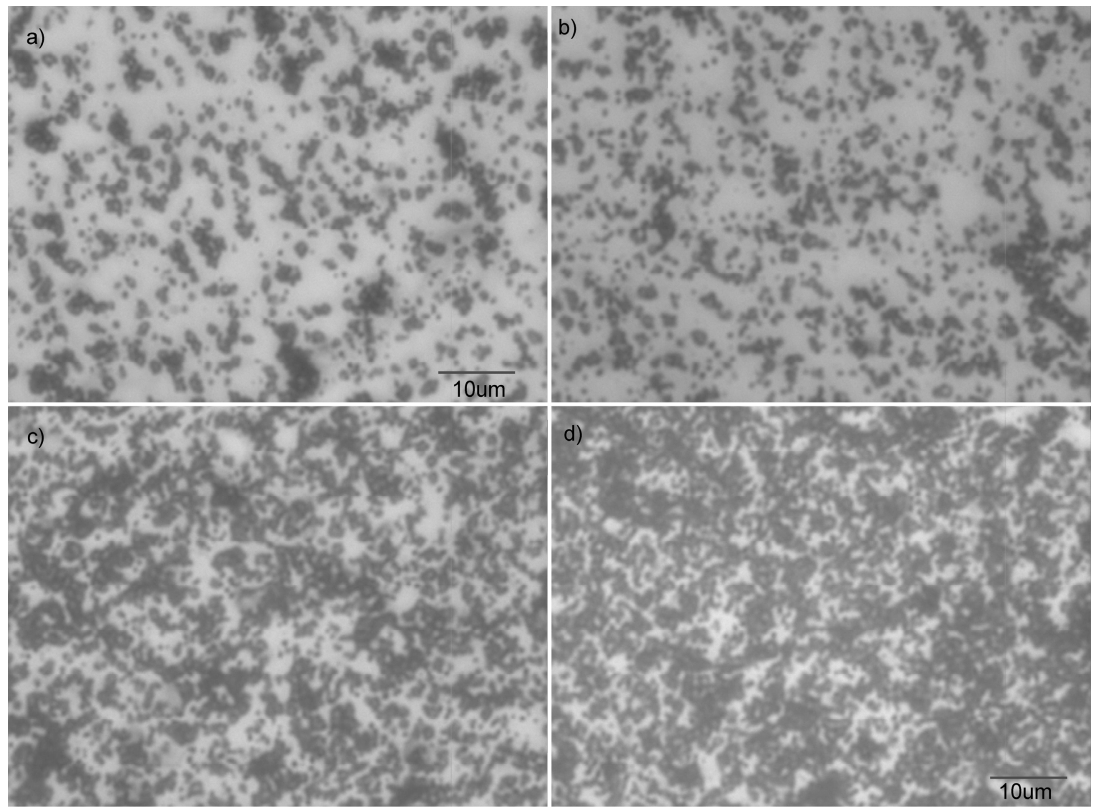

Figure 3: Optical micrographs of thin sections of TPE/PZT composite films containing a) $50 \mathrm{wt} \%$ b) $60 \mathrm{wt} \%$ c) $70 \mathrm{wt} \%$ d) $80 \mathrm{wt} \%$ PZT.

The materials properties of the TPE/PZT composites at different filler concentrations are shown in figure 4 relative to those of the matrix material. The dielectric constant increased with increasing filler content such that at $80 \mathrm{wt} \%$ filler there was a more than threefold increase in $\varepsilon$. There was a similar increase of the modulus determined from tensile tests at $0 \%$ strain so that a concentration of $80 \mathrm{wt} \%$ filler again led to a nearly threefold increase. The modulus determined at $30 \%$ strain, which lies within the plateau region of the stress-strain curve of the matrix, was less strongly influenced by the filler content, as noted previously for the TPE/PZT composites fabricated via melt extrusion. This behavior may be accounted for by the formation of a network of strongly interacting particles which breaks down at higher strains. Prestraining the composite films may therefore be beneficial in terms of actuation performance.

The increase in the permittivity can be well described by Brüggeman's mixing rule ${ }^{17}$

$$
\frac{\varepsilon_{f}-\varepsilon_{c o m p}}{\varepsilon_{f}-\varepsilon_{m}} \sqrt[3]{\frac{\varepsilon_{m}}{\varepsilon_{c o m p}}}=1-\phi \quad \text { (Equation 3) }
$$

where $\varepsilon_{\mathrm{i}}$ with $i=m, f$, comp is the permittivity of the matrix, filler, and composite respectively, and $\phi$ the volume content of the filler. The model curve taking 2 as permittivity of the matrix and 1725 for the PZT is shown as solid line in Figure 4. By this model one is able to predict the permittivity of composites containing higher filler levels. At the limiting value of $63.4 \mathrm{vol} \%$ for the closest random package of spheres ${ }^{18}$, a value of 38.3 would be expected meaning a 19 fold increase compared to the TPE. Unfortunately, higher filler levels resulted in materials behaving more as a highly viscous paste and less as an elastomer. Thus, such materials might be of interest for flexible electronics, particularly as all composites showed strains in excess of $1000 \%$ in tensile tests, but are of less value for DEA. 


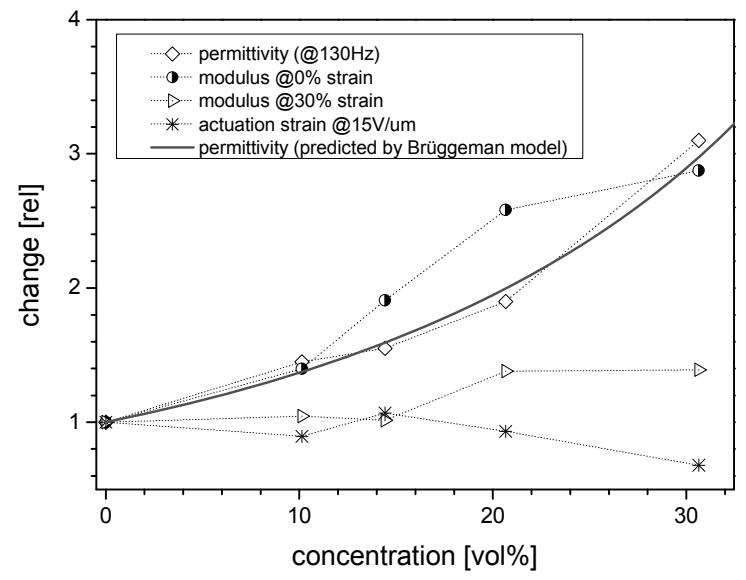

Figure 4: Variation in materials parameters for TPE/PZT composites normalized with respect to the corresponding values for the TPE matrix, which are $112 \mathrm{kPa}$ and $96 \mathrm{kPa}$ for the modulus at $0 \%$ and $30 \%$ respectively, 2 for the permittivity, and $1.34 \%$ strain at $15 \mathrm{~V} / \mu \mathrm{m}$. Solid line is the predicted permittivity according to Brüggeman's mixing rule.

The electromechanical response of composites derived from TPE/PZT composites with different amounts of filler was measured using circular actuators. Results for the composites and the neat TPE are shown in Figure 5. The electric field $E=V / d$ was calculated from the nominal electric field $E=V / d_{0}$, and the reduced thickness assuming incompressibility, $d=d_{0}(s+1)^{-2}$ of the material, where $s$ is the measured in-plane linear strain and $d_{0}$ is the initial thickness. Although the dielectric and mechanical properties changed significantly on filler addition, only small changes in the actuation strain were observed at a given $E$. On the other hand, based on equation 1 , an increase in actuation stress is expected for a given strain, although a direct stress measurement was not possible in the chosen actuator configuration.

\begin{tabular}{|l|c|c|c|c|c|}
\hline PZT concentration & $0 \mathrm{wt} \%$ & $50 \mathrm{wt} \%$ & $60 \mathrm{wt} \%$ & $70 \mathrm{wt} \%$ & $80 \mathrm{wt} \%$ \\
\hline $\begin{array}{l}\text { breakdown field in } \\
\text { actuator }(\mathrm{V} / \mu \mathrm{m})\end{array}$ & 50.0 & 34.3 & 28.8 & 25.0 & 20.6 \\
\hline
\end{tabular}

Table 1: Electrical breakdown field of actuators made from TPE/PZT composites with different PZT concentrations

As shown in table 1, the electrical breakdown field was significantly modified in composites containing PZT, decreasing monotonically with increasing filler content. This clearly limits the maximum energy conversion that can be achieved with any DEA produced from such composites. The reason for such behavior is not clear although it may originate from ion conductivity at high electric field strengths or from electromechanical instabilities ${ }^{19}$, such that the electrostatic pressure increases more rapidly than the strain hardening of the elastomer. 


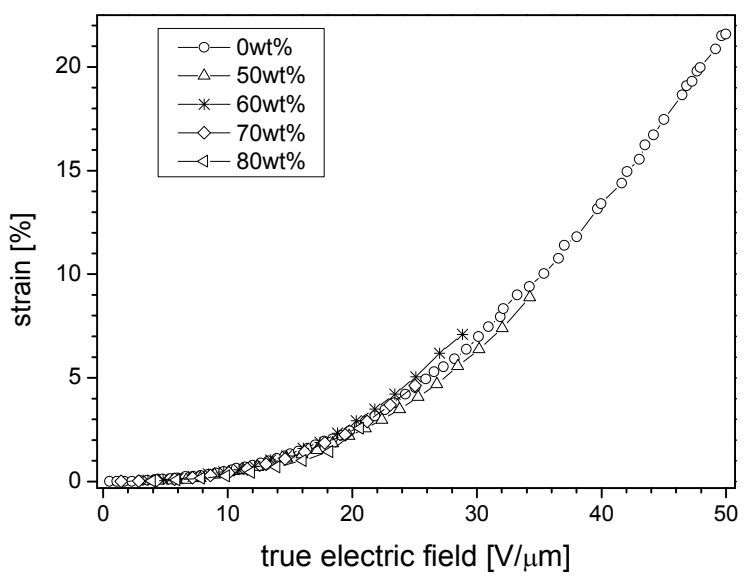

Figure 5: Observed actuation strain for composites containing different amounts of ceramic filler compared to that of the neat TPE matrix. The electrical breakdown strength of the composite actuators was significantly reduced compared to that of the TPE matrix, values are given in table 1.

From dynamical mechanical analysis, which was performed in shear mode, it was observed that not only the elastic modulus changes with filler concentration but that this is also accompanied by an increase in the mechanical loss factor, $\tan \delta$ (i.e. the ratio of the imaginary to the real part of the complex shear modulus) whereas the frequency dependency of $\tan \delta$ is reduced. This behavior is depicted in Figure 6, the higher mechanical loss clearly represents a further limitation on the efficiency of DEAs produced from such materials. ${ }^{20}$ The frequency dependence of $\tan \delta$ clearly

Finally, the density of ceramic filler composites increases linearly with the filler volume content from $0.87 \mathrm{~g} / \mathrm{cm}^{3}$ for the TPE to $6.22 \mathrm{~g} / \mathrm{cm}^{3}$ for the composite containing $80 \mathrm{wt} \%$ of PZT, so that the weight saving advantages of low density polymers are progressively lost.

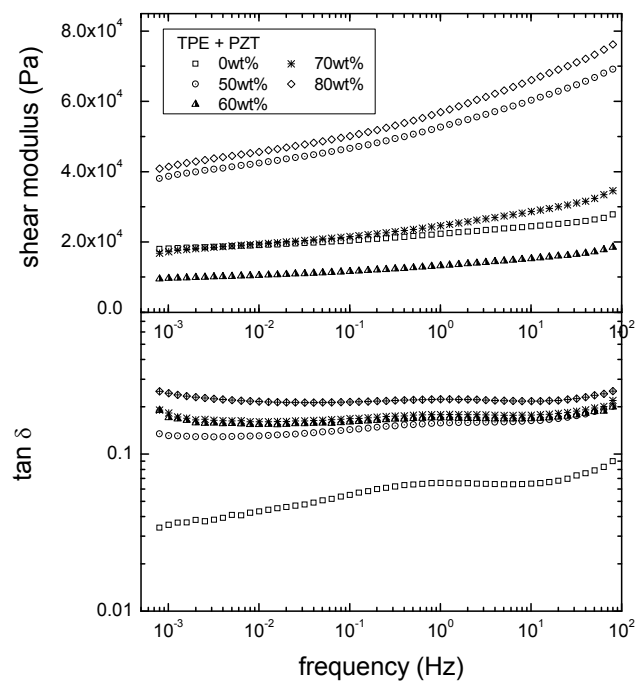

Figure 6: Shear modulus (storage modulus) and mechanical loss factor tan $\delta$ obtained from oscillatory shear tests on TPE/PZT composites with different filler concentration. 


\section{CONCLUSIONS \& OUTLOOK}

Composites prepared from a thermoplastic elastomer showed high permittivity in the presence of ceramic or conductive fillers. However, no significant increase in performance of actuation was obtained in either composite system, although the ceramic filler composites may show enhanced performance in terms of the mechanical stress achievable at a given electric field. The increase in the dielectric constant was accompanied by a drastic increase in electric conductivity of the TPE/PANI composite and an increase in density and modulus of elasticity of the TPE/PZT composites. Controlling the process parameters made it possible to tailor the dispersion of PZT particles in the matrix, but this had relatively little effect on the electromagnetic response of the resulting materials.

A possible way to limit the increases in electrical conductivity is to use conducting particles encapsulated in an insulating shell, allowing precise control of the minimum interparticle distance even in the presence of agglomeration, and disrupting conductive paths. We have recently ${ }^{21}$ developed a procedure for encapsulating PANI particles in an insulating shell and are currently investigation the usefulness of this approach.

Regardless of the trends in electrical conductivity, the present results suggest that increases in the dielectric constant at high filler contents are offset by increases in matrix stiffness. Indeed, in a recent investigation of three different elastomer matrices, the frequency dependence of the actuation performance has been shown to be dominated by the mechanical response rather than the dielectric properties. ${ }^{22}$ It is therefore suggested that the mechanical properties should also provide the focus for future systematic materials development in this area.

\section{AKNOWLEGEMENT}

Financial Support by Empa (Project No. 841331) is kindly acknowledged. The author is thankful to Dr. Gabor Kovacs for the opportunity to use the high-voltage setup and Christian Walder for the DMA measurements. Christa Jordi and Arne Schmidt are thanked for helpful discussions.

\section{REFERENCES}

[1] Bar-Cohen, Y. and Zhang, Q. "Electroactive polymer actuators and sensors", MRS Bulletin 33, 173-181 (2008)

[2] Carpi, F., Rossi, D. D., Kornbluh, R., Pelrine, R. and Sommer-Larsen, P., [Dielectric Elastomers as Electromechanical Transducers], Elsevier (2008)

[3] Carpi, F., Gallone, G., Galantini, F. and de Rossi, D., "Silicone-Poly(hexyl-thiophene blends as elastomers with enhanced electromechanical transduction properties)", Adv. Func. Mat. 18, 235-241 (2008)

[4] Gallone, G., Carpi, F., Galantini, F., de Rossi, D. and Levita, G., "Enhancing the electro-mechanical response of Maxwell stress actuators", Adv. in Sci. and Tech. 61, 46-53 (2008)

[5] Gallone, G., Carpi, F., de Rossi, D., Levita, G. and Marchetti, A., "Dielectric constant enhancement in a silicone elastomer filled with lead magnesium niobate-lead titanate", Mater. Sci.\& Eng. C 27, 110-116 (2007)

[6] Mc Carthy, D. N., Risse, S., Katekomol, P. and Kofod, G., "The effect of dispersion on the increased relative permittivity of TiO2/SEBS composites", J. of Phys. D 42(14), 145406 (2009)

[7] Zhang, X., Löwe, C., Wissler, M., Jähne, B. and Kovacs, G. "Dielectric elastomers in actuator technology" Adv. Eng. Mater. 7, 361-367 (2005)

[8] Huang, C. and Zhang, Q., "Enhanced dielectric and electromechanical responses in high dielectric constant allpolymer percolative composites", Adv. Func. Mater. 14(5), 501-506 (2004)

[9] Pelrine, R., Kornbluh, R. and Joseph, J., "Electrostriction of polymer dielectrics with compliant electrodes as a means of actuation", Sens. \& Act. A 64(1), 77-85 (1998)

[10] Patsidis, A. and Psarras, G. C., "Dielectric behaviour and functionality of polymer matrix - ceramic $\mathrm{BaTiO}_{3}$ composites", Express Polymer Letters 2(10), 718-726 (2008)

[11] Dang, Z.-M., Wang, L., Yin, Y., Zhang, Q. and Lei, Q.-Q. "Giant dielectric permittivities in functionalized carbonnanotubes/electroactive-polymer nanocomposites" Adv. Mater. 19, 852-857 (2007) 
[12] Qi, L., Samuels, W. D., Exarhos, G. J. and Parler, S. G., "Three-phase percolative silver-BaTiO3-Epoxy nanocomposite with dielectric constants", J. Appl. Pol. Sci. 102, 967-971 (2006)

[13] Lim, E.-S., Lee, J.-C., Kim, J.-J., Park, E.-T., Chung, Y.-K. and Lee, H.-Y., "Dielectric characteristic of polymerceramic-metal composites for the application of embedded passive devices", Int. Ferroelectrics 74, 53-60 (2005)

[14] Wilkinson, D., Langer, J. S. and Sen, P. N., "Enhancement of the dielectric constant near a percolation threshold", Physical Review B 28(2), 1081-1087 (1983)

[15] Stoyanov, H., Mc Carthy, D., Kollosche, M. and Kofod, G., "Dielectric properties and electric breakdown strength of a subpercolative composite of carbon black in thermoplastic copolymer", Appl. Phys Lett. 94(23), 232905 (2009)

[16] Walder, C., Molberg, M., Opris, D. M., Nüesch, F. A., Löwe, C., Plummer, C. J. G. , Leterrier, Y., Manson, J-A. E. "High k dielectric elastomeric materials for low voltage applications", Proc of SPIE 7287, 72870Q (2009)

[17] Brüggeman, D. A. G., "Berechnung verschiedener physikalischer Konstanten von heterogenen Substanzen I. Dielektrizitätskonstanten und Leitfähigkeiten der Mischkörper aus isotropen Substanzen", Ann. Phys. 24, 636 (1935)

[18] Song, C.; Wang, P., Makse, H. A., "A phase diagram for jammed matter", Nature 453, 629 (2008)

[19] Zhao, X. H. and Suo, Z. G. "Electromechanical instability in semicrystalline polymers" Appl. Phys. Lett. 95, 031904 (2009)

[20] Palakodeti, R. and Kessler, M. R., "Influence of frequency and prestrain on the mechanical efficiency of dielectric electroactive polymer actuators" Mater Lett., 60, 3437-3440 (2006)

[21] Opris, D. M., Löwe, C. , Molberg, M., Crespy, D., Nüesch, F. A., "Phthalocyanine and encapsulated polyaniline nanoparticles as fillers for dielectric elastomers" Proc of SPIE 7287, 72870L (2009)

[22] Molberg, M., Leterrier, Y., Plummer, C. J. G., Walder, C., Löwe, C., Opris, D. M., Nüesch, F. A., Bauer, S. and Manson, J.-A. E. "Frequency dependent dielectric and mechanical behavior of elastomers for actuator applications" J. Appl. Phys. 106, 054112 (2009) 